\title{
Article \\ A Certain Subclass of Multivalent Analytic Functions Defined by the $q$-Difference Operator Related to the Janowski Functions
}

\author{
Bo Wang ${ }^{1}$, Rekha Srivastava ${ }^{2, *}$ (1) and Jin-Lin Liu ${ }^{1}$ \\ 1 Department of Mathematics, Yangzhou University, Yangzhou 225002, China; bowangYZU@163.com (B.W.); \\ jlliu@yzu.edu.cn (J.-L.L.) \\ 2 Department of Mathematics and Statistics, University of Victoria, Victoria, BC V8W 3R4, Canada \\ * Correspondence: rekhas@math.uvic.ca
}

check for updates

Citation: Wang, B.; Srivastava, R.; Liu, J.-L. A Certain Subclass of Multivalent Analytic Functions Defined by the $q$-Difference Operator Related to the Janowski Functions. Mathematics 2021, 9, 1706. https:// doi.org/10.3390/math9141706

Academic Editor: Abdelmejid Bayad

Received: 20 June 2021

Accepted: 8 July 2021

Published: 20 July 2021

Publisher's Note: MDPI stays neutral with regard to jurisdictional claims in published maps and institutional affiliations.

Copyright: (c) 2021 by the authors. Licensee MDPI, Basel, Switzerland. This article is an open access article distributed under the terms and conditions of the Creative Commons Attribution (CC BY) license (https:// creativecommons.org/licenses/by/ $4.0 /)$.
Abstract: A class of $p$-valent analytic functions is introduced using the $q$-difference operator and the familiar Janowski functions. Several properties of functions in the class, such as the Fekete-Szegö inequality, coefficient estimates, necessary and sufficient conditions, distortion and growth theorems, radii of convexity and starlikeness, closure theorems and partial sums, are discussed in this paper.

Keywords: analytic functions; $q$-difference operator; univalent and multivalent functions; Janowski functions; Fekete-Szegö inequality; distortion theorem; radii of starlikeness and convexity; partial sum; closure theorems

\section{Introduction}

The $q$-calculus is classical calculus without the concept of limit. In recent years, $q$ calculus has attracted great attention of scholars on account of its applications in the research field of physics and mathematics as, for example, in the study of quantum groups, $q$-deformed superalgebras, fractals and multifractal measures, optimal control problems and in chaotic dynamical systems. The application of $q$-calculus involving $q$-derivatives and $q$-integrals was initiated by Jackson [1,2]. Later, the $q$-derivative operator (or $q$-difference operator) was used to investigate the geometry of $q$-starlike functions for the first time in [3]. Moreover, Aral [4] and Anastassiou and Gal [5,6] generalized some complex operators which are known as the $q$-Picad and the $q$-Gauss-Weierstrass singular integral operators. Recently, Srivastava et al. [7] have written a series of articles [8-10] in which they combined the $q$-difference operator and the Janowski functions to define new function classes and studied their useful properties from different viewpoints. In addition, we choose to refer the interested reader to further developments on $q$-theory in [11-18]. In paricular, in his recent survey-cum-expository review article, Srivastava [18] exposed the trivial and inconsequential developments in the literature in which known $q$-results are being routinely translated into the corresponding $(\mathfrak{p}, q)$-results by forcing an obviously redundant or superfluous parameter $\mathfrak{p}$ into the known $q$-results.

Let $A_{p}$ denote the class of $p$-valent analytic functions $f(z)$ given by the following Taylor-Maclaurin series expansion:

$$
f(z)=z^{p}+\sum_{n=1}^{\infty} a_{p+n} z^{p+n} \quad(p \in \mathbb{N})
$$

in the open unit disk $D=\{z:|z|<1\}$. For $p=1$, we write $A:=A_{1}$. In the whole paper, we let $\mathbb{N}, \mathbb{C}$ and $\mathbb{R}$ be the sets of positive integers, complex numbers and real numbers, respectively.

A function $f(z) \in A_{p}$ is called to be a $p$-valent starlike function of order $\delta$ and is written as $f(z) \in S_{p}^{*}(\delta)$, if it satisfies the following inequality:

$$
\operatorname{Re}\left(\frac{z f^{\prime}(z)}{f(z)}\right)>\delta \quad(0 \leqq \delta<p)
$$


for all $z \in D$.

A function $f(z) \in A_{p}$ is known as a $p$-valent convex function of order $\delta$ and is denoted by $f(z) \in C_{p}(\delta)$, if it meets the following condition:

$$
\operatorname{Re}\left(1+\frac{z f^{\prime \prime}(z)}{f^{\prime}(z)}\right)>\delta \quad(0 \leqq \delta<p)
$$

for all $z \in D$.

From (1) and (2), we have the following equivalence:

$$
f(z) \in C_{p}(\delta) \Leftrightarrow \frac{z f^{\prime}(z)}{p} \in S_{p}^{*}(\delta) .
$$

Definition 1. Let $0<q<1$ and introduce the $q$-number $[\lambda]_{q}$ by

$$
[\lambda]_{q}= \begin{cases}\frac{1-q^{\lambda}}{1-q} & (\lambda \in \mathbb{C}) \\ \sum_{k=0}^{n-1} q^{k}=1+q+q^{2}+\cdots+q^{n-1} & (\lambda=n \in \mathbb{N}) .\end{cases}
$$

Let $[\lambda]_{q} \cdot[\lambda]_{q}:=[\lambda]_{q}^{2}$. In particular, when $\lambda=0$, we have $[0]_{q}=0$.

Definition 2 (See $[1,2])$. Let $0<q<1$. Then the $q$-difference operator $D_{q}$ of a function $f(z)$ is given by

$$
D_{q} f(z)= \begin{cases}\frac{f(z)-f(q z)}{(1-q) z} & (z \neq 0) \\ f^{\prime}(0) & (z=0),\end{cases}
$$

if $f^{\prime}(0)$ exists.

One can observe from Definition 2 that

$$
\lim _{q \rightarrow 1-} D_{q} f(z)=\lim _{q \rightarrow 1-} \frac{f(q z)-f(z)}{(q-1) z}=f^{\prime}(z),
$$

provided that $f(z)$ is a differentiable function in a set of $\mathbb{C}$. Furthermore, for $f(z)=z^{p}+$ $\sum_{n=1}^{\infty} a_{p+n} z^{p+n}$, one can see that

$$
D_{q} f(z)=[p]_{q} z^{p-1}+\sum_{n=1}^{\infty}[p+n]_{q} a_{p+n} z^{p+n-1} \quad(z \neq 0),
$$

where

$$
[p]_{q}=\frac{1-q^{p}}{1-q}=1+q+q^{2}+\cdots+q^{p-1} .
$$

A function $f(z)$ belonging to $A_{p}$ is called to be a p-valent $q$-starlike function of order $\sigma$ and is written as $f(z) \in S_{p, q}^{*}(\sigma)$, if it meets the condition:

$$
\operatorname{Re}\left(\frac{z D_{q} f(z)}{f(z)}\right)>\sigma \quad\left(0 \leqq \sigma<[p]_{q}\right)
$$

for all $z \in D$.

A function $f(z)$ belonging to $A_{p}$ is referred to as a $p$-valent $q$-convex function of order $\sigma$ and is written as $f(z) \in C_{p, q}(\sigma)$, if it meets the condition:

$$
\operatorname{Re}\left(\frac{D_{q}\left(z D_{q} f(z)\right)}{D_{q} f(z)}\right)>\sigma \quad\left(0 \leqq \sigma<[p]_{q}\right)
$$


for all $z \in D$.

From (3) and (4), it is not difficult to verify that

$$
f(z) \in C_{p, q}(\sigma) \Leftrightarrow \frac{z D_{q} f(z)}{[p]_{q}} \in S_{p, q}^{*}(\sigma) .
$$

Definition 3. A function $h(z)$, analytic in $D$ with $h(0)=1$, is called to belong to $P[A, B]$, if

$$
h(z) \prec \frac{1+A z}{1+B z} \quad(-1 \leqq B<A \leqq 1),
$$

equivalently we can write

$$
\left|\frac{h(z)-1}{A-B h(z)}\right|<1
$$

For analytic functions $h(z)$ and $p(z)(z \in D)$, the function $p(z)$ is said to subordinate to the function $h(z)$ and written $p(z) \prec h(z)(z \in D)$, if there exists an analytic function $w(z)(z \in D)$ with $w(0)=0$ and $|w(z)|<1$ so that $p(z)=h(w(z))(z \in D)$. Suppose that $h(z)$ is analytic univalent in $D$, then the following equivalence holds true:

$$
p(z) \prec h(z) \quad(z \in D) \Longleftrightarrow p(0)=h(0) \quad \text { and } \quad p(D) \subset h(D) .
$$

In $q$-calculus concept, we now define the following subclasses of $A_{p}$ in connection with the q-difference operator $D_{q}$.

Definition 4. A function $f(z)$ belonging to $A_{p}$ is called to be in $I_{p, q}(\alpha, A, B)$, if it meets the condition $\frac{1}{1-\alpha}\left(\frac{z D_{q} f(z)}{[p]_{q} f(z)}-\alpha \frac{z D_{q}\left(z D_{q} f(z)\right)}{[p]_{q}^{2} f(z)}\right) \prec \frac{1+A z}{1+B z}, \quad-1 \leqq B<A \leqq 1, \quad 0 \leqq \alpha<1, \quad q \in(0,1)$, or equivalently

$$
\left|\frac{\frac{z D_{q} f(z)}{[p]_{q} f(z)}-\alpha \frac{z D_{q}\left(z D_{q} f(z)\right)}{[p]_{q}^{2} f(z)}-(1-\alpha)}{(1-\alpha) A-B\left(\frac{z D_{q} f(z)}{[p]_{q} f(z)}-\alpha \frac{z D_{q}\left(z D_{q} f(z)\right)}{[p]_{q}^{2} f(z)}\right)}\right|<1
$$

We note that:

(1) For $\alpha=0$, we obtain $S_{p, q}^{*}[A, B]$, the family of $p$-valent $q$-starlike functions associated with Janowski function;

(2) For $\alpha=0, A=1$ and $B=-1$, we obtain $S_{p, q}^{*}$, the family of $p$-valent $q$-starlike functions;

(3) For $\alpha=0, A=1, B=-1$ and $q \rightarrow 1-$, we have $S_{p}^{*}$, the family of $p$-valent starlike functions;

(4) For $\alpha=0, A=1, B=-1, q \rightarrow 1$ - and $p=1$, we obtain $S^{*}$, the family of starlike functions.

A well-known question in GFT is to discuss the functional composed of combinations of certain coefficients of functions. The class $A_{1}$ is made up of functions of the form $f(z)=z+$ $a_{2} z^{2}+a_{3} z^{3}+\cdots(z \in D)$. The Fekete-Szegö functional describes a specific relationship between coefficient $a_{2}$ and $a_{3}$, i.e., $\left|a_{3}-\lambda a_{2}^{2}\right|, 0 \leqq \lambda \leqq 1$. Fekete and Szegö [19] found that $\left|a_{3}-\lambda a_{2}^{2}\right|$ is bounded by $1+2 \exp (-\lambda /(1-\lambda))$ for $0 \leqq \lambda<1$ and $f(z) \in A_{1}$ and the bound is sharp for every $\lambda$. In particular, if we let $f(z) \in A_{1}$ and $\lambda=1$, then $\left|a_{3}-a_{2}^{2}\right| \leqq 1$. More recently, Srivastava et al. researched the Fekete-Szegö inequalities for several classes of $q$-convex and $q$-starlike functions in [20].

Let $\Omega$ denote the family of functions of the form:

$$
w(z)=w_{1} z+w_{2} z^{2}+w_{3} z^{3}+\cdots,
$$

in $D$ with $|w(z)|<1$. 
To derive the main results, we recall the following lemmas.

Lemma 1 (References [21-23]). Let $w(z) \in \Omega$. Then

$$
\left|w_{2}-t w_{1}^{2}\right| \leqq \max \{1,|t|\}= \begin{cases}-t & (t \leqq-1) \\ 1 & (-1 \leqq t \leqq 1) \\ t & (t \geqq 1)\end{cases}
$$

For $t>1$ or $t<-1$, the equality occurs when $w(z)=z$ or $w(z)=z e^{i \theta}$. For $t \in(-1,1)$, the equality is true when $w(z)=z^{2}$ or $w(z)=z^{2} e^{i \theta}$. For $t=-1$, the equality occurs when

$$
w(z)=\frac{(\eta+z) z}{\eta z+1} \quad(0 \leqq \eta \leqq 1) .
$$

For $t=1$, the equality occurs when

$$
w(z)=-\frac{(\eta+z) z}{\eta z+1} \quad(0 \leqq \eta \leqq 1) .
$$

These above upper bounds are best possible, and they could be further extended. For $t \in(-1,1)$ :

$$
\left|w_{2}-t w_{1}^{2}\right|+(t+1)\left|w_{1}\right|^{2} \leqq 1 \quad(t \in(-1,0]),
$$

and

$$
\left|w_{2}-t w_{1}^{2}\right|+(1-t)\left|w_{1}\right|^{2} \leqq 1 \quad(t \in(0,1)) .
$$

Lemma 2 (Reference [24]). Let $w(z) \in \Omega$. Then for $q_{1}, q_{2} \in \mathbb{R}$, we have the following sharp estimates:

$$
H\left(q_{1}, q_{2}\right) \geq\left|q_{1} w_{1} w_{2}+q_{2} w_{1}^{3}+w_{3}\right|,
$$

where

$$
H\left(q_{1}, q_{2}\right)= \begin{cases}1 & \left(q_{1}, q_{2}\right) \in D_{1} \cup D_{2} \\ \left|q_{2}\right| & \left(q_{1}, q_{2}\right) \in \cup_{k=3}^{7} D_{k} \\ \frac{2}{3}\left(1+\left|q_{1}\right|\right)\left(\frac{1+\left|q_{1}\right|}{3\left(1+\left|q_{1}\right|+q_{2}\right)}\right)^{\frac{1}{2}} & \left(q_{1}, q_{2}\right) \in D_{8} \cup D_{9} \\ \frac{q_{2}}{3}\left(\frac{q_{1}^{2}-4}{q_{1}^{2}-4 q_{2}}\right)\left(\frac{q_{1}^{2}-4}{3\left(q_{2}-1\right)}\right)^{\frac{1}{2}} & \left(q_{1}, q_{2}\right) \in D_{10} \cup D_{11}-\{ \pm 2,1\} \\ \frac{2}{3}\left(\left|q_{1}\right|-1\right)\left(\frac{1-\left|q_{1}\right|}{3\left(1-\left|q_{1}\right|+q_{2}\right)}\right)^{\frac{1}{2}} & \left(q_{1}, q_{2}\right) \in D_{12} .\end{cases}
$$


The above $D_{k}(k=1,2, \cdots, 12)$ are given as the following:

$$
\begin{aligned}
& D_{1}=\left\{\left(q_{1}, q_{2}\right):\left|q_{1}\right| \leqq \frac{1}{2},\left|q_{2}\right| \leqq 1\right\} \text {, } \\
& D_{2}=\left\{\left(q_{1}, q_{2}\right): \frac{1}{2} \leqq\left|q_{1}\right| \leqq 2, \frac{4}{27}\left(1+\left|q_{1}\right|\right)^{3}-\left(1+\left|q_{1}\right|\right) \leqq q_{2} \leqq 1\right\} \text {, } \\
& D_{3}=\left\{\left(q_{1}, q_{2}\right):\left|q_{1}\right| \leqq \frac{1}{2}, q_{2} \leqq-1\right\} \text {, } \\
& D_{4}=\left\{\left(q_{1}, q_{2}\right):\left|q_{1}\right| \geqq \frac{1}{2}, q_{2} \leqq-\frac{2}{3}\left(1+\left|q_{1}\right|\right)\right\}, \\
& D_{5}=\left\{\left(q_{1}, q_{2}\right):\left|q_{1}\right| \leqq 2, q_{2} \geqq 1\right\} \text {, } \\
& D_{6}=\left\{\left(q_{1}, q_{2}\right): 2 \leqq\left|q_{1}\right| \leqq 4, q_{2} \geqq \frac{1}{12}\left(8+q_{1}^{2}\right)\right\} \text {, } \\
& D_{7}=\left\{\left(q_{1}, q_{2}\right):\left|q_{1}\right| \geqq 4, q_{2} \geqq \frac{2}{3}\left(\left|q_{1}\right|-1\right)\right\} \text {, } \\
& D_{8}=\left\{\left(q_{1}, q_{2}\right): \frac{1}{2} \leqq\left|q_{1}\right| \leqq 2,-\frac{2}{3}\left(1+\left|q_{1}\right|\right) \leqq q_{2} \leqq \frac{4}{27}\left(1+\left|q_{1}\right|\right)^{3}-\left(1+\left|q_{1}\right|\right)\right\}, \\
& D_{9}=\left\{\left(q_{1}, q_{2}\right):\left|q_{1}\right| \geqq 2,-\frac{2}{3}\left(1+\left|q_{1}\right|\right) \leqq q_{2} \leqq \frac{2\left|q_{1}\right|\left(1+\left|q_{1}\right|\right)}{q_{1}^{2}+2\left|q_{1}\right|+4}\right\}, \\
& D_{10}=\left\{\left(q_{1}, q_{2}\right): 2 \leqq\left|q_{1}\right| \leqq 4, \frac{2\left|q_{1}\right|\left(1+\left|q_{1}\right|\right)}{4+q_{1}^{2}+2\left|q_{1}\right|} \leqq q_{2} \leqq \frac{1}{12}\left(8+q_{1}^{2}\right)\right\} \text {, } \\
& D_{11}=\left\{\left(q_{1}, q_{2}\right):\left|q_{1}\right| \geqq 4, \frac{2\left|q_{1}\right|\left(1+\left|q_{1}\right|\right)}{4+q_{1}^{2}+2\left|q_{1}\right|} \leqq q_{2} \leqq \frac{2\left|q_{1}\right|\left(\left|q_{1}\right|-1\right)}{4+q_{1}^{2}-2\left|q_{1}\right|}\right\} \text {, } \\
& D_{12}=\left\{\left(q_{1}, q_{2}\right):\left|q_{1}\right| \geqq 4, \frac{2\left|q_{1}\right|\left(\left|q_{1}\right|-1\right)}{4+q_{1}^{2}-2\left|q_{1}\right|} \leqq q_{2} \leqq \frac{2}{3}\left(\left|q_{1}\right|-1\right)\right\} \text {. }
\end{aligned}
$$

Unless otherwise stated, we assume the entire paper that

$$
-1 \leqq B<A \leqq 1,0 \leqq \alpha<1 \text { and } q \in(0,1) \text {. }
$$

In this paper, we shall study some geometric properties of functions belonging to $I_{p, q}(\alpha, A, B)$ such as Fekete-Szegö inequality, necessary and sufficient conditions, distortion and growth theorems, coefficient estimates, radii of convexity and starlikeness, closure theorems and partial sums.

\section{Main Results}

Theorem 1. Let $f(z) \in A_{p}$ and

$$
0 \leqq \alpha \leqq \frac{[p+1]_{q}[p]_{q}-[p]_{q}^{2}}{[p+3]_{q}^{2}-[p]_{q}^{2}}
$$

If $f(z) \in I_{p, q}(\alpha, A, B)$ and $\mu \in \mathbb{C}$, then

$$
\left|a_{p+2}-\mu a_{p+1}^{2}\right| \leqq \frac{(1-\alpha)(A-B)[p]_{q}^{2}}{E_{2}} \max \left\{1,\left|B+\frac{(1-\alpha)(A-B)[p]_{q}^{2}}{E_{1}}\left(\mu \frac{E_{2}}{E_{1}}-1\right)\right|\right\}
$$

and

$$
\left|a_{p+3}\right| \leqq \frac{(1-\alpha)(A-B)[p]_{q}^{2}}{E_{3}} H\left(q_{1}, q_{2}\right)
$$


where

$$
\begin{aligned}
E_{n} & =[p+n]_{q}\left([p]_{q}-\alpha[p+n]_{q}\right)-(1-\alpha)[p]_{q}^{2} \quad(n=1,2,3), \\
q_{1} & =-2 B+\frac{(1-\alpha)(A-B)[p]_{q}^{2}\left(E_{1}+E_{2}\right)}{E_{1} E_{2}}, \\
q_{2} & =B^{2}-\left(\frac{(1-\alpha)(A-B)[p]_{q}^{2}}{E_{1}}\right)^{2} \\
& +\frac{(1-\alpha)(A-B)[p]_{q}^{2}\left(E_{1}+E_{2}\right)}{E_{1} E_{2}}\left(-B+\frac{(1-\alpha)(A-B)[p]_{q}^{2}}{E_{1}}\right) .
\end{aligned}
$$

Proof of Theorem 1. If $f(z) \in I_{p, q}(\alpha, A, B)$, by Definition 4 , there is a function

$$
w(z)=w_{1} z+w_{2} z^{2}+w_{3} z^{3}+\cdots \in \Omega,
$$

such that

$$
\frac{1}{1-\alpha}\left(\frac{z D_{q} f(z)}{[p]_{q} f(z)}-\alpha \frac{z D_{q}\left(z D_{q} f(z)\right)}{[p]_{q}^{2} f(z)}\right)=\frac{1+A w(z)}{1+B w(z)} .
$$

Since

$$
\begin{aligned}
\frac{1}{1-\alpha}\left(\frac{z D_{q} f(z)}{[p]_{q} f(z)}-\alpha \frac{z D_{q}\left(z D_{q} f(z)\right)}{[p]_{q}^{2} f(z)}\right)= & 1+\frac{E_{1}}{(1-\alpha)[p]_{q}^{2}} a_{p+1} z+ \\
& \left(\frac{E_{2}}{(1-\alpha)[p]_{q}^{2}} a_{p+2}-\frac{E_{1}}{(1-\alpha)[p]_{q}^{2}} a_{p+1}^{2}\right) z^{2}+ \\
& \left(\frac{E_{3}}{(1-\alpha)[p]_{q}^{2}} a_{p+3}+\frac{E_{1}}{(1-\alpha)[p]_{q}^{2}} a_{p+1}^{3}-\right. \\
& \left.\frac{E_{1}+E_{2}}{(1-\alpha)[p]_{q}^{2}} a_{p+1} a_{p+2}\right) z^{3}+\cdots
\end{aligned}
$$

and

$$
\frac{1+A w(z)}{1+B w(z)}=1+(A-B) w_{1} z+(A-B)\left(w_{2}-B w_{1}^{2}\right) z^{2}+(A-B)\left(w_{3}-2 B w_{1} w_{2}+B^{2} w_{1}^{3}\right) z^{3}+\cdots,
$$

we have

$$
\begin{aligned}
a_{p+1}= & \frac{(1-\alpha)(A-B)[p]_{q}^{2} w_{1}}{E_{1}} \\
a_{p+2}= & \frac{(1-\alpha)(A-B)[p]_{q}^{2}}{E_{2}}\left[w_{2}+w_{1}^{2}\left(-B+\frac{(1-\alpha)(A-B)[p]_{q}^{2}}{E_{1}}\right)\right] \\
a_{p+3}= & \frac{(1-\alpha)(A-B)[p]_{q}^{2}}{E_{3}}\left\{w_{3}+\left(-2 B+\frac{(1-\alpha)(A-B)[p]_{q}^{2}\left(E_{1}+E_{2}\right)}{E_{1} E_{2}}\right) w_{1} w_{2}+\right. \\
& {\left[B^{2}-\left(\frac{(1-\alpha)(A-B)[p]_{q}^{2}}{E_{1}}\right)^{2}+\frac{(1-\alpha)(A-B)[p]_{q}^{2}\left(E_{1}+E_{2}\right)}{E_{1} E_{2}} .\right.} \\
& {\left.\left[-B+\left(\frac{(1-\alpha)(A-B)[p]_{q}^{2}}{E_{1}}\right)\right]\right\} . }
\end{aligned}
$$




\section{Therefore, we obtain}

$$
a_{p+2}-\mu a_{p+1}^{2}=\frac{(1-\alpha)(A-B)[p]_{q}^{2}}{E_{2}}\left\{w_{2}-v w_{1}^{2}\right\}
$$

where

$$
v=B+\frac{(1-\alpha)(A-B)[p]_{q}^{2}}{E_{1}}\left(\mu \frac{E_{2}}{E_{1}}-1\right)
$$

By applying Lemmas 1 and 2 to (8) and (9), respectively, we obtain (6) and (7). Now the proof of the Theorem is completed.

Corollary 1. Let $f(z) \in A_{p}$ and $0 \leqq \alpha \leqq \frac{[p+1]_{q}[p]_{q}-[p]_{q}^{2}}{[p+3]_{q}^{2}-[p]_{q}^{2}}$. If $f(z) \in I_{p, q}(\alpha, A, B)$ and $\mu \in \mathbb{R}$, then

$$
\left|a_{p+2}-\mu a_{p+1}^{2}\right| \leqq \begin{cases}\frac{(1-\alpha)(A-B)[p]_{q}^{2}}{E_{2}}\left(-B+\frac{(1-\alpha)(A-B)[p]_{q}^{2}}{E_{1}}\left(1-\mu \frac{E_{2}}{E_{1}}\right)\right) & \left(\mu \leqq \sigma_{1}\right) \\ \frac{(1-\alpha)(A-B)[p]_{q}^{2}}{E_{2}} & \left(\sigma_{1} \leqq \mu \leqq \sigma_{2}\right) \\ \frac{(1-\alpha)(A-B)[p]_{q}^{2}}{E_{2}}\left(B+\frac{(1-\alpha)(A-B)[p]_{q}^{2}}{E_{1}}\left(\mu \frac{E_{2}}{E_{1}}-1\right)\right) & \left(\mu \geqq \sigma_{2}\right),\end{cases}
$$

where

$$
\sigma_{1}=\frac{\left[(-1-B) E_{1}+(1-\alpha)(A-B)[p]_{q}^{2}\right] E_{1}}{(1-\alpha)(A-B)[p]_{q}^{2} E_{2}}
$$

and

$$
\sigma_{2}=\frac{\left[(1-B) E_{1}+(1-\alpha)(A-B)[p]_{q}^{2}\right] E_{1}}{(1-\alpha)(A-B)[p]_{q}^{2} E_{2}}
$$

Furthermore, let

$$
\sigma_{3}=\frac{\sigma_{1}+\sigma_{2}}{2}=\frac{\left[-B E_{1}+(1-\alpha)(A-B)[p]_{q}^{2}\right] E_{1}}{(1-\alpha)(A-B)[p]_{q}^{2} E_{2}}
$$

If $\sigma_{1}<\mu \leqq \sigma_{3}$, then

$$
\begin{gathered}
\left|a_{p+2}-\mu a_{p+1}^{2}\right|+\frac{E_{1}^{2}}{(1-\alpha)(A-B)[p]_{q}^{2} E_{2}}\left[(1+B)+\frac{(1-\alpha)(A-B)[p]_{q}^{2}}{E_{1}} .\right. \\
\left.\left(\mu \frac{E_{2}}{E_{1}}-1\right)\right]\left|a_{p+1}\right|^{2} \leqq \frac{(1-\alpha)(A-B)[p]_{q}^{2}}{E_{2}} .
\end{gathered}
$$

If $\sigma_{3}<\mu<\sigma_{2}$, then

$$
\begin{gathered}
\left|a_{p+2}-\mu a_{p+1}^{2}\right|+\frac{E_{1}^{2}}{(1-\alpha)(A-B)[p]_{q}^{2} E_{2}}\left[(1-B)+\frac{(1-\alpha)(A-B)[p]_{q}^{2}}{E_{1}} .\right. \\
\left.\left(1-\mu \frac{E_{2}}{E_{1}}\right)\right]\left|a_{p+1}\right|^{2} \leqq \frac{(1-\alpha)(A-B)[p]_{q}^{2}}{E_{2}} .
\end{gathered}
$$

Theorem 2. Let $\frac{[p]_{q}}{[p+1]_{q}} \leqq \alpha<1$. Additionally, let

$$
f(z)=z^{p}-\sum_{n=1}^{\infty}\left|a_{p+n}\right| z^{p+n} \in A_{p} .
$$


Then the function $f(z)$ belongs to $I_{p, q}(\alpha, A, B)$ if and only if

$$
\begin{aligned}
& \sum_{n=1}^{\infty}\left((1+B)[n+p]_{q}\left(\alpha[n+p]_{q}-[p]_{q}\right)+(1-\alpha)(1+A)[p]_{q}^{2}\right)\left|a_{p+n}\right| \\
& \leqq(1-\alpha)(A-B)[p]_{q}^{2} .
\end{aligned}
$$

Proof of Theorem 2. Assuming that the inequality (10) holds true, we need to show the inequality (5). Now we have

$$
\begin{aligned}
& \left|\frac{\frac{z D_{q} f(z)}{[p]_{q} f(z)}-\alpha \frac{z D_{q}\left(z D_{q} f(z)\right)}{[p]_{q}^{2} f(z)}-(1-\alpha)}{(1-\alpha) A-B\left(\frac{z D_{q} f(z)}{[p]_{q} f(z)}-\alpha \frac{z D_{q}\left(z D_{q} f(z)\right)}{[p]_{q}^{2} f(z)}\right)}\right| \\
& =\left|\frac{[p]_{q} z D_{q} f(z)-\alpha z D_{q}\left(z D_{q} f(z)\right)-(1-\alpha)[p]_{q}^{2} f(z)}{(1-\alpha) A[p]_{q}^{2} f(z)-B\left([p]_{q} z D_{q} f(z)-\alpha z D_{q}\left(z D_{q} f(z)\right)\right)}\right| \\
& =\left|\frac{\sum_{n=1}^{\infty}\left([n+p]_{q}\left(\alpha[n+p]_{q}-[p]_{q}\right)+(1-\alpha)[p]_{q}^{2}\right)\left|a_{n+p}\right| z^{n+p}}{(A-B)(1-\alpha)[p]_{q}^{2} z^{p}-\sum_{n=1}^{\infty}\left(B[n+p]_{q}\left(\alpha[n+p]_{q}-[p]_{q}\right)+(1-\alpha) A[p]_{q}^{2}\right)\left|a_{n+p}\right| z^{n+p}}\right| \\
& =\left|\frac{\sum_{n=1}^{\infty}\left([n+p]_{q}\left(\alpha[n+p]_{q}-[p]_{q}\right)+(1-\alpha)[p]_{q}^{2}\right)\left|a_{n+p}\right| z^{n}}{(A-B)(1-\alpha)[p]_{q}^{2}-\sum_{n=1}^{\infty}\left(B[n+p]_{q}\left(\alpha[n+p]_{q}-[p]_{q}\right)+(1-\alpha) A[p]_{q}^{2}\right)\left|a_{n+p}\right| z^{n}}\right|<1,
\end{aligned}
$$

which shows that the function $f(z)$ belongs to $I_{p, q}(\alpha, A, B)$.

On the other hand, we let the function $f(z)=z^{p}-\sum_{n=1}^{\infty}\left|a_{p+n}\right| z^{p+n} \in I_{p, q}(\alpha, A, B)$. Then from (5), one can see that

$$
\begin{aligned}
& \left|\frac{\frac{z D_{q} f(z)}{[p]_{q} f(z)}-\alpha \frac{z D_{q}\left(z D_{q} f(z)\right)}{[p]_{q}^{2} f(z)}-(1-\alpha)}{(1-\alpha) A-B\left(\frac{z D_{q} f(z)}{[p]_{q} f(z)}-\alpha \frac{z D_{q}\left(z D_{q} f(z)\right)}{[p]_{q}^{2} f(z)}\right)}\right| \\
& =\left|\frac{\sum_{n=1}^{\infty}\left([p+n]_{q}\left(\alpha[p+n]_{q}-[p]_{q}\right)+(1-\alpha)[p]_{q}^{2}\right)\left|a_{p+n}\right| z^{n}}{(1-\alpha)(A-B)[p]_{q}^{2}-\sum_{n=1}^{\infty}\left(B[p+n]_{q}\left(\alpha[p+n]_{q}-[p]_{q}\right)+(1-\alpha) A[p]_{q}^{2}\right)\left|a_{p+n}\right| z^{n}}\right| \\
& <1 \text {. }
\end{aligned}
$$

The inequality (11) is correct for $z \in D$. By choosing $z=\operatorname{Re} z \rightarrow 1$, we obtain (10). Thus, the Theorem is proved.

Corollary 2. Let $\frac{[p]_{q}}{[p+1]_{q}} \leqq \alpha<1$. If $f(z)=z^{p}-\sum_{n=1}^{\infty}\left|a_{p+n}\right| z^{p+n} \in I_{p, q}(\alpha, A, B)$, then

$$
\left|a_{n+p}\right| \leqq \frac{(A-B)(1-\alpha)[p]_{q}^{2}}{(1+B)[n+p]_{q}\left(\alpha[n+p]_{q}-[p]_{q}\right)+(1-\alpha)(1+A)[p]_{q}^{2}} \quad(n=1,2, \cdots) .
$$

The result is best possible for $f(z)$ defined as

$$
f(z)=z^{p}-\frac{(A-B)(1-\alpha)[p]_{q}^{2}}{(1+B)[n+p]_{q}\left(\alpha[n+p]_{q}-[p]_{q}\right)+(1+A)(1-\alpha)[p]_{q}^{2}} z^{n+p} \quad(n=1,2, \cdots) .
$$

Theorem 3. Let $\frac{[p]_{q}}{[p+1]_{q}} \leqq \alpha<1$. If

$$
f(z)=z^{p}-\sum_{n=1}^{\infty}\left|a_{p+n}\right| z^{p+n} \in I_{p, q}(\alpha, A, B),
$$

then, for $|z|=r<1$,

$$
r^{p}-\tau_{1} r^{p+1} \leqq|f(z)| \leqq r^{p}+\tau_{1} r^{p+1},
$$


where

$$
\tau_{1}=\frac{(A-B)(1-\alpha)[p]_{q}^{2}}{(B+1)[1+p]_{q}\left(\alpha[1+p]_{q}-[p]_{q}\right)+(1+A)(1-\alpha)[p]_{q}^{2}} .
$$

The bounds are best possible for $f(z)$ given as

$$
f(z)=z^{p}-\frac{(A-B)(1-\alpha)[p]_{q}^{2}}{(B+1)[1+p]_{q}\left(\alpha[1+p]_{q}-[p]_{q}\right)+(1+A)(1-\alpha)[p]_{q}^{2}} z^{1+p} .
$$

Proof of Theorem 3. Let

$$
f(z)=z^{p}-\sum_{n=1}^{\infty}\left|a_{p+n}\right| z^{p+n} .
$$

Then, by applying the triangle inequality, we have

$$
|f(z)|=\left|z^{p}-\sum_{n=1}^{\infty}\right| a_{p+n}\left|z^{p+n}\right| \leqq|z|^{p}+\sum_{n=1}^{\infty}\left|a_{p+n}\right||z|^{p+n} .
$$

Since $|z|=r<1$, we can see that $r^{p+n} \leqq r^{p+1}$. Thus, we have

$$
|f(z)| \leqq r^{p}+r^{p+1} \sum_{n=1}^{\infty}\left|a_{p+n}\right|
$$

and

$$
|f(z)| \geqq r^{p}-r^{p+1} \sum_{n=1}^{\infty}\left|a_{p+n}\right| .
$$

Considering $f(z) \in I_{p, q}(\alpha, A, B)$, we know from Theorem 2 that

$$
\begin{aligned}
& \sum_{n=1}^{\infty}\left((1+B)[n+p]_{q}\left(\alpha[n+p]_{q}-[p]_{q}\right)+(1+A)(1-\alpha)[p]_{q}^{2}\right)\left|a_{n+p}\right| \\
& \leqq(A-B)(1-\alpha)[p]_{q}^{2} .
\end{aligned}
$$

Since the sequence $\left\{(1+B)[n+p]_{q}\left(\alpha[n+p]_{q}-[p]_{q}\right)+(1+A)(1-\alpha)[p]_{q}^{2}\right\}$ is increasing regarding $n(n \geqq 1)$, we have

$$
\begin{aligned}
& \left((1+B)[1+p]_{q}\left(\alpha[1+p]_{q}-[p]_{q}\right)+(1+A)(1-\alpha)[p]_{q}^{2}\right) \sum_{n=1}^{\infty}\left|a_{n+p}\right| \\
& \leqq \sum_{n=1}^{\infty}\left((B+1)[n+p]_{q}\left(\alpha[n+p]_{q}-[p]_{q}\right)+(1+A)(1-\alpha)[p]_{q}^{2}\right)\left|a_{n+p}\right| .
\end{aligned}
$$

Hence by transitivity we obtain

$\left((B+1)[1+p]_{q}\left(\alpha[1+p]_{q}-[p]_{q}\right)+(1+A)(1-\alpha)[p]_{q}^{2}\right) \sum_{n=1}^{\infty}\left|a_{n+p}\right| \leqq(A-B)(1-\alpha)[p]_{q}^{2}$,

which implies that

$$
\sum_{n=1}^{\infty}\left|a_{n+p}\right| \leqq \frac{(A-B)(1-\alpha)[p]_{q}^{2}}{(B+1)[1+p]_{q}\left(\alpha[1+p]_{q}-[p]_{q}\right)+(1+A)(1-\alpha)[p]_{q}^{2}}
$$

Substituting (14) into (12) and (13), we obtain the required results. The proof of Theorem 3 is completed.

Theorem 4. Let $\frac{[p]_{q}}{[p+1]_{q}} \leqq \alpha<1$ and $\frac{(1+A)[p]_{q}\left([p+1]_{q}-[p]_{q}\right)-[p+1]_{q}^{2}}{[p+1]_{q}^{2}}<B<A \leqq 1$. If $f(z)=$ $z^{p}-\sum_{n=1}^{\infty}\left|a_{p+n}\right| z^{p+n} \in I_{p, q}(\alpha, A, B)$, then, for $|z|=r<1$, we have

$$
[p]_{q} r^{p-1}-\tau_{2} r^{p} \leqq\left|D_{q} f(z)\right| \leqq[p]_{q} r^{p-1}+\tau_{2} r^{p}
$$

where

$$
\tau_{2}=\frac{(A-B)(1-\alpha)[p]_{q}^{2}[1+p]_{q}}{(B+1)[1+p]_{q}\left(\alpha[1+p]_{q}-[p]_{q}\right)+(1+A)(1-\alpha)[p]_{q}^{2}}
$$


The results are best possible for the following function

$$
f(z)=z^{p}-\frac{(A-B)(1-\alpha)[p]_{q}^{2}}{(B+1)[1+p]_{q}\left(\alpha[1+p]_{q}-[p]_{q}\right)+(1+A)(1-\alpha)[p]_{q}^{2}} z^{1+p} .
$$

Proof of Theorem 4. Let

$$
f(z)=z^{p}-\sum_{n=1}^{\infty}\left|a_{p+n}\right| z^{p+n} .
$$

Then, from Definition 2, we can write

$$
D_{q} f(z)=[p]_{q} z^{p-1}-\sum_{n=1}^{\infty}[p+n]_{q}\left|a_{p+n}\right| z^{p+n-1} .
$$

By applying the triangle inequality, we obtain

$$
\left|D_{q} f(z)\right|=\left|[p]_{q} z^{p-1}-\sum_{n=1}^{\infty}[n+p]_{q}\right| a_{n+p}\left|z^{n+p-1}\right| \leqq[p]_{q}|z|^{p-1}+\sum_{n=1}^{\infty}[n+p]_{q}\left|a_{n+p}\right||z|^{n+p-1} .
$$

Furthermore, we have

$$
\left|D_{q} f(z)\right| \leqq r^{p-1}[p]_{q}+r^{p} \sum_{n=1}^{\infty}[n+p]_{q}\left|a_{n+p}\right|
$$

and

$$
\left|D_{q} f(z)\right| \geqq r^{p-1}[p]_{q}-r^{p} \sum_{n=1}^{\infty}[n+p]_{q}\left|a_{n+p}\right| .
$$

Because of the function $f(z)$ belonging to the class $I_{p, q}(\alpha, A, B)$, we find from Theorem 2 that

$$
\sum_{n=1}^{\infty}\left((B+1)\left(\alpha[n+p]_{q}-[p]_{q}\right)+\frac{(1+A)(1-\alpha)[p]_{q}^{2}}{[n+p]_{q}}\right)[n+p]_{q}\left|a_{n+p}\right| \leqq(A-B)(1-\alpha)[p]_{q}^{2} .
$$

As we know that

$$
\left\{(1+B)\left(\alpha[p+n]_{q}-[p]_{q}\right)+\frac{(1-\alpha)(1+A)[p]_{q}^{2}}{[p+n]_{q}}\right\}
$$

is an increasing sequence regarding $n(n \geqq 1)$, so

$$
\begin{aligned}
& \left((B+1)\left(\alpha[1+p]_{q}-[p]_{q}\right)+\frac{(1+A)(1-\alpha)[p]_{q}^{2}}{[1+p]_{q}}\right) \sum_{n=1}^{\infty}[n+p]_{q}\left|a_{n+p}\right| \\
& \leqq \sum_{n=1}^{\infty}\left((B+1)\left(\alpha[n+p]_{q}-[p]_{q}\right)+\frac{(1+A)(1-\alpha)[p]_{q}^{2}}{[n+p]_{q}}\right)[n+p]_{q}\left|a_{n+p}\right| .
\end{aligned}
$$

Thus, by transitivity, we have

$$
\left((B+1)\left(\alpha[1+p]_{q}-[p]_{q}\right)+\frac{(1+A)(1-\alpha)[p]_{q}^{2}}{[1+p]_{q}}\right) \sum_{n=1}^{\infty}[n+p]_{q}\left|a_{n+p}\right| \leqq(A-B)(1-\alpha)[p]_{q}^{2},
$$

which implies that

$$
\sum_{n=1}^{\infty}[n+p]_{q}\left|a_{n+p}\right| \leqq \frac{(A-B)(1-\alpha)[p]_{q}^{2}[1+p]_{q}}{(B+1)[1+p]_{q}\left(\alpha[1+p]_{q}-[p]_{q}\right)+(1+A)(1-\alpha)[p]_{q}^{2}} .
$$

Now, by putting (17) in (15) and (16), we complete the proof of Theorem 4.

Theorem 5. Let

$$
\frac{[p]_{q}}{[p+1]_{q}} \leqq \alpha<1 \quad\left(-1 \leqq B_{2}<0<B_{1} \leqq 1\right)
$$


Additionally, let $0 \leqq \delta<p$. If

$$
f(z)=z^{p}-\sum_{n=1}^{\infty}\left|a_{p+n}\right| z^{p+n} \in I_{p, q}(\alpha, A, B),
$$

then, for $0<|z|<r_{1}, f(z)$ is p-valent starlike function of order $\delta$, where

$r_{1}=\min \left\{\inf _{n \geqq 1}\left(\frac{(p-\delta)\left(B_{1}-B_{2}\right)\left((B+1)[n+p]_{q}\left(\alpha[n+p]_{q}-[p]_{q}\right)+(1+A)(1-\alpha)[p]_{q}^{2}\right)}{\left((p-\delta)\left(B_{1}-B_{2}\right)+n\left(1-B_{2}\right)\right)(A-B)(1-\alpha)[p]_{q}^{2}}\right)^{\frac{1}{n}}, 1\right\}$.

Proof of Theorem 5. Let $f(z) \in I_{p, q}(\alpha, A, B)$. In order to prove $f(z) \in S_{p}^{*}(\delta)$, we need to show that

$$
\frac{\frac{z f^{\prime}(z)}{f(z)}-\delta}{p-\delta} \prec \frac{1+B_{1} z}{1+B_{2} z}, \quad\left(0 \leqq \delta<p,-1 \leqq B_{2}<0<B_{1} \leqq 1\right) .
$$

The subordination above is equivalent to $\left|\frac{z f^{\prime}(z)-p f(z)}{-B_{2} z f^{\prime}(z)+\left(p B_{1}-\delta\left(B_{1}-B_{2}\right)\right) f(z)}\right|<1$. After some calculations and simplifications, we obtain

$$
\sum_{n=1}^{\infty} \frac{(p-\delta)\left(B_{1}-B_{2}\right)+n\left(1-B_{2}\right)}{(p-\delta)\left(B_{1}-B_{2}\right)}\left|a_{p+n}\right||z|^{n}<1 .
$$

From the inequality (10), we can obviously find that

$$
\sum_{n=1}^{\infty} \frac{(B+1)[n+p]_{q}\left(\alpha[n+p]_{q}-[p]_{q}\right)+(1+A)(1-\alpha)[p]_{q}^{2}}{(A-B)(1-\alpha)[p]_{q}^{2}}\left|a_{n+p}\right|<1 .
$$

Inequality (18) can be seen to be true if it satisfies the following inequality:

$$
\begin{aligned}
& \sum_{n=1}^{\infty} \frac{(p-\delta)\left(B_{1}-B_{2}\right)+n\left(1-B_{2}\right)}{(p-\delta)\left(B_{1}-B_{2}\right)}\left|a_{p+n}\right||z|^{n} \\
& <\sum_{n=1}^{\infty} \frac{(1+B)[p+n]_{q}\left(\alpha[p+n]_{q}-[p]_{q}\right)+(1-\alpha)(1+A)[p]_{q}^{2}}{(1-\alpha)(A-B)[p]_{q}^{2}}\left|a_{p+n}\right| .
\end{aligned}
$$

The above inequality indicates that

$$
|z|^{n}<\frac{(p-\delta)\left(B_{1}-B_{2}\right)\left((B+1)[n+p]_{q}\left(\alpha[n+p]_{q}-[p]_{q}\right)+(1+A)(1-\alpha)[p]_{q}^{2}\right)}{\left((p-\delta)\left(B_{1}-B_{2}\right)+n\left(1-B_{2}\right)\right)(A-B)(1-\alpha)[p]_{q}^{2}}
$$

or

$$
|z|<\left(\frac{(p-\delta)\left(B_{1}-B_{2}\right)\left((1+B)[p+n]_{q}\left(\alpha[p+n]_{q}-[p]_{q}\right)+(1-\alpha)(1+A)[p]_{q}^{2}\right)}{\left((p-\delta)\left(B_{1}-B_{2}\right)+n\left(1-B_{2}\right)\right)(1-\alpha)(A-B)[p]_{q}^{2}}\right)^{\frac{1}{n}} .
$$

Let

$$
r_{1}=\min \left\{\inf _{n \geqq 1}\left(\frac{(p-\delta)\left(B_{1}-B_{2}\right)\left((B+1)[n+p]_{q}\left(\alpha[n+p]_{q}-[p]_{q}\right)+(1+A)(1-\alpha)[p]_{q}^{2}\right)}{\left((p-\delta)\left(B_{1}-B_{2}\right)+n\left(1-B_{2}\right)\right)(A-B)(1-\alpha)[p]_{q}^{2}}\right)^{\frac{1}{n}}, 1\right\},
$$

then we obtain the required result. The proof of Theorem 5 is completed.

With the aid of the method in the proof of Theorem 5, we also obtain the following theorems for the classes $C_{p}(\delta), S_{p, q}^{*}(\sigma)$ and $C_{p, q}(\sigma)$, respectively. 
Theorem 6. Let $\frac{[p]_{q}}{[p+1]_{q}} \leqq \alpha<1,-1 \leqq B_{2}<0<B_{1} \leqq 1$ and $0 \leqq \delta<p$. If

$$
f(z)=z^{p}-\sum_{n=1}^{\infty}\left|a_{p+n}\right| z^{p+n} \in I_{p, q}(\alpha, A, B),
$$

then, for $0<|z|<r_{2}, f(z)$ is p-valent convex function of order $\delta$, where

$$
r_{2}=\min \left\{\inf _{n \geqq 1}\left(\frac{p(p-\delta)\left(B_{1}-B_{2}\right)\left((B+1)[n+p]_{q}\left(\alpha[n+p]_{q}-[p]_{q}\right)+(1+A)(1-\alpha)[p]_{q}^{2}\right)}{(n+p)\left((p-\delta)\left(B_{1}-B_{2}\right)+n\left(1-B_{2}\right)\right)(A-B)(1-\alpha)[p]_{q}^{2}}\right)^{\frac{1}{n}}, 1\right\} .
$$

Theorem 7. Let $\frac{[p]_{q}}{[p+1]_{q}} \leqq \alpha<1,-1 \leqq B_{2}<0<B_{1} \leqq 1$ and $0 \leqq \sigma<[p]_{q}$. If

$$
f(z)=z^{p}-\sum_{n=1}^{\infty}\left|a_{p+n}\right| z^{p+n} \in I_{p, q}(\alpha, A, B),
$$

then, for $0<|z|<r_{3}, f(z)$ is $p$-valent $q$-starlike function of order $\sigma$, where

$$
r_{3}=\min \left\{\inf _{n \geqq 1}\left(\frac{\left([p]_{q}-\sigma\right)\left(B_{1}-B_{2}\right)\left((B+1)[n+p]_{q}\left(\alpha[n+p]_{q}-[p]_{q}\right)+(1+A)(1-\alpha)[p]_{q}^{2}\right)}{\left([n+p]_{q}\left(1-B_{2}\right)-[p]_{q}\left(1-B_{1}\right)-\sigma\left(B_{1}-B_{2}\right)\right)(A-B)(1-\alpha)[p]_{q}^{2}}\right)^{\frac{1}{n}}, 1\right\} .
$$

Theorem 8. Let $\frac{[p]_{q}}{[p+1]_{q}} \leqq \alpha<1,0 \leqq \sigma<[p]_{q}$ and $-1 \leqq B_{2}<0<B_{1} \leqq 1$. If the function

$$
f(z)=z^{p}-\sum_{n=1}^{\infty}\left|a_{n+p}\right| z^{n+p} \in I_{p, q}(\alpha, A, B),
$$

then, for $0<|z|<r_{4}, f(z)$ is p-valent $q$-convex function of order $\sigma$, where

$r_{4}=\min \left\{\inf _{n \geqq 1}\left(\frac{[p]_{q}\left([p]_{q}-\sigma\right)\left(B_{1}-B_{2}\right)\left((B+1)[n+p]_{q}\left(\alpha[n+p]_{q}-[p]_{q}\right)+(1+A)(1-\alpha)[p]_{q}^{2}\right)}{[n+p]_{q}\left([n+p]_{q}\left(1-B_{2}\right)-[p]_{q}\left(1-B_{1}\right)-\sigma\left(B_{1}-B_{2}\right)\right)(A-B)(1-\alpha)[p]_{q}^{2}}\right)^{\frac{1}{n}}, 1\right\}$.

Taking $B_{1}=1$ and $B_{2}=-1$ in Theorems 5 to 8 , we obtain the following corollaries, respectively.

Corollary 3. Let $0 \leqq \delta<p$ and $\frac{[p]_{q}}{[p+1]_{q}} \leqq \alpha<1$. If the function

$$
f(z)=z^{p}-\sum_{n=1}^{\infty}\left|a_{n+p}\right| z^{n+p} \in I_{p, q}(\alpha, A, B),
$$

then, for $0<|z|<r_{5}, f(z)$ is p-valent starlike function of order $\delta$, where

$$
r_{5}=\min \left\{\inf _{n \geqq 1}\left(\frac{(p-\delta)\left((B+1)[n+p]_{q}\left(\alpha[n+p]_{q}-[p]_{q}\right)+(1+A)(1-\alpha)[p]_{q}^{2}\right)}{(A-B)(1-\alpha)(n+p-\delta)[p]_{q}^{2}}\right)^{\frac{1}{n}}, 1\right\} .
$$

Corollary 4. Let $0 \leqq \delta<p$ and $\frac{[p]_{q}}{[p+1]_{q}} \leqq \alpha<1$. If the function

$$
f(z)=z^{p}-\sum_{n=1}^{\infty}\left|a_{n+p}\right| z^{n+p} \in I_{p, q}(\alpha, A, B),
$$


then, for $0<|z|<r_{6}, f(z)$ is $p$-valent convex function of order $\delta$, where

$$
r_{6}=\min \left\{\inf _{n \geqq 1}\left(\frac{p(p-\delta)\left((B+1)[n+p]_{q}\left(\alpha[n+p]_{q}-[p]_{q}\right)+(1+A)(1-\alpha)[p]_{q}^{2}\right)}{(A-B)(1-\alpha)(n+p)(n+p-\delta)[p]_{q}^{2}}\right)^{\frac{1}{n}}, 1\right\} .
$$

Corollary 5. Let $0 \leqq \sigma<[p]_{q}$ and $\frac{[p]_{q}}{[p+1]_{q}} \leqq \alpha<1$. If the function

$$
f(z)=z^{p}-\sum_{n=1}^{\infty}\left|a_{n+p}\right| z^{n+p} \in I_{p, q}(\alpha, A, B),
$$

then, for $0<|z|<r_{7}, f(z)$ is p-valent $q$-starlike function of order $\sigma$, where

$$
r_{7}=\min \left\{\inf _{n \geqq 1}\left(\frac{\left([p]_{q}-\sigma\right)\left((B+1)[n+p]_{q}\left(\alpha[n+p]_{q}-[p]_{q}\right)+(1+A)(1-\alpha)[p]_{q}^{2}\right)}{\left([n+p]_{q}-\sigma\right)(A-B)(1-\alpha)[p]_{q}^{2}}\right)^{\frac{1}{n}}, 1\right\} .
$$

Corollary 6. Let $0 \leqq \sigma<[p]_{q}$ and $\frac{[p]_{q}}{[p+1]_{q}} \leqq \alpha<1$. If the function

$$
f(z)=z^{p}-\sum_{n=1}^{\infty}\left|a_{n+p}\right| z^{n+p} \in I_{p, q}(\alpha, A, B),
$$

then, for $0<|z|<r_{8}, f(z)$ is $p$-valent $q$-convex function of order $\sigma$, where

$$
r_{8}=\min \left\{\inf _{n \geqq 1}\left(\frac{[p]_{q}\left([p]_{q}-\sigma\right)\left((B+1)[n+p]_{q}\left(\alpha[n+p]_{q}-[p]_{q}\right)+(1+A)(1-\alpha)[p]_{q}^{2}\right)}{[n+p]_{q}\left([n+p]_{q}-\sigma\right)(A-B)(1-\alpha)[p]_{q}^{2}}\right)^{\frac{1}{n}}, 1\right\} .
$$

Next, we will study the ratio of a function

$$
f(z)=z^{p}-\sum_{n=1}^{\infty}\left|a_{p+n}\right| z^{p+n}
$$

to its sequence of partial sums given by

$$
f_{k}(z)=z^{p}-\sum_{n=1}^{\infty}\left|a_{p+n}\right| z^{p+n} \quad(\forall z \in D) .
$$

Theorem 9. Let $\frac{[p]_{q}}{[p+1]_{q}} \leqq \alpha<1$. If

$$
f(z)=z^{p}-\sum_{n=1}^{\infty}\left|a_{p+n}\right| z^{p+n} \in I_{p, q}(\alpha, A, B),
$$

then

$$
\operatorname{Re}\left(\frac{f(z)}{f_{k}(z)}\right)>1-\frac{1}{\varphi_{k+1}} \quad(z \in D)
$$

and

$$
\operatorname{Re}\left(\frac{f_{k}(z)}{f(z)}\right)>\frac{\varphi_{k+1}}{1+\varphi_{k+1}} \quad(z \in D)
$$

where

$$
\varphi_{k+1}=\frac{(B+1)[1+p+k]_{q}\left(\alpha[1+p+k]_{q}-[p]_{q}\right)+(1+A)(1-\alpha)[p]_{q}^{2}}{(A-B)(1-\alpha)[p]_{q}^{2}}
$$

Proof of Theorem 9. To prove (19), we set

$$
\varphi_{k+1}\left[\frac{f(z)}{f_{k}(z)}-\left(1-\frac{1}{\varphi_{k+1}}\right)\right]=\frac{1-\sum_{n=1}^{k}\left|a_{p+n}\right| z^{n}-\varphi_{k+1} \sum_{n=k+1}^{\infty}\left|a_{p+n}\right| z^{n}}{1-\sum_{n=1}^{k}\left|a_{p+n}\right| z^{n}}=\frac{1+w(z)}{1-w(z)} .
$$


After some simplifications, we have

$$
w(z)=\frac{-\varphi_{k+1} \sum_{n=k+1}^{\infty}\left|a_{p+n}\right| z^{n}}{2-2 \sum_{n=1}^{k}\left|a_{p+n}\right| z^{n}-\varphi_{k+1} \sum_{n=k+1}^{\infty}\left|a_{p+n}\right| z^{n}}
$$

and

$$
|w(z)| \leqq \frac{\varphi_{k+1} \sum_{n=k+1}^{\infty}\left|a_{p+n}\right|}{2-2 \sum_{n=1}^{k}\left|a_{p+n}\right|-\varphi_{k+1} \sum_{n=k+1}^{\infty}\left|a_{p+n}\right|} .
$$

Thus, we find that $|w(z)|<1$ if and only if

$$
\sum_{n=1}^{k}\left|a_{p+n}\right|+\varphi_{k+1} \sum_{n=k+1}^{\infty}\left|a_{p+n}\right|<1 .
$$

From (10) we have

$$
\sum_{n=1}^{\infty} \varphi_{n}\left|a_{n+p}\right|<1 .
$$

It is not difficult to see that the sequence $\left\{\varphi_{n}\right\}$ is increasing regarding $n$. Additionally, we can see that $\varphi_{n} \geqq 1(n=1,2, \cdots)$. Thus, we obtain

$$
\sum_{n=1}^{k}\left|a_{n+p}\right|+\varphi_{k+1} \sum_{n=k+1}^{\infty}\left|a_{n+p}\right| \leqq \sum_{n=1}^{k} \varphi_{n}\left|a_{n+p}\right|+\sum_{n=k+1}^{\infty} \varphi_{n}\left|a_{n+p}\right|=\sum_{n=1}^{\infty} \varphi_{n}\left|a_{n+p}\right|<1 .
$$

Thus, the inequality (22) is true. This proves (19).

Next, in order to prove the inequality (20), we consider

$$
\left(1+\varphi_{k+1}\right)\left[\frac{f_{k}(z)}{f(z)}-\frac{\varphi_{k+1}}{1+\varphi_{k+1}}\right]=\frac{1-\sum_{n=1}^{k}\left|a_{p+n}\right| z^{n}+\varphi_{k+1} \sum_{n=k+1}^{\infty}\left|a_{p+n}\right| z^{n}}{1-\sum_{n=1}^{\infty}\left|a_{p+n}\right| z^{n}}=\frac{1+w(z)}{1-w(z)} .
$$

By simple calculating, we find that

$$
w(z)=\frac{\left(1+\varphi_{k+1}\right) \sum_{n=k+1}^{\infty}\left|a_{n+p}\right| z^{n}}{2+\left(\varphi_{k+1}-1\right) \sum_{n=k+1}^{\infty}\left|a_{n+p}\right| z^{n}-2 \sum_{n=1}^{k}\left|a_{n+p}\right| z^{n}}
$$

and

$$
|w(z)| \leqq \frac{\left(1+\varphi_{k+1}\right) \sum_{n=k+1}^{\infty}\left|a_{n+p}\right|}{2-\left(\varphi_{k+1}-1\right) \sum_{n=k+1}^{\infty}\left|a_{n+p}\right|-2 \sum_{n=1}^{k}\left|a_{n+p}\right|} .
$$

Thus, we obtain $|w(z)|<1$ if it satisfies the following condition:

$$
\sum_{n=1}^{k}\left|a_{p+n}\right|+\varphi_{k+1} \sum_{n=k+1}^{\infty}\left|a_{p+n}\right|<1 .
$$

The remaining part of the proof is similar to that of (19) and we omit it. Now we complete The proof of Theorem 9 .

Theorem 10. Let $\frac{[p]_{q}}{[p+1]_{q}} \leqq \alpha<1$. If

$$
f_{j}(z)=z^{p}-\sum_{n=1}^{\infty}\left|a_{p+n, j}\right| z^{p+n} \in I_{p, q}(\alpha, A, B) \quad(j=1,2),
$$

then, for $0 \leqq \lambda \leqq 1$, the function

$$
H_{\lambda}(z)=\lambda f_{1}(z)+(1-\lambda) f_{2}(z) \in I_{p, q}(\alpha, A, B) .
$$

Proof of Theorem 10. For $0 \leqq \lambda \leqq 1$, the function $H_{\lambda}(z)$ can be written as follows:

$$
H_{\lambda}(z)=\lambda f_{1}(z)+(1-\lambda) f_{2}(z)=z^{p}-\sum_{n=1}^{\infty}\left(\lambda\left|a_{n+p, 1}\right|+(1-\lambda)\left|a_{n+p, 2}\right|\right) z^{n+p} .
$$


For functions $f_{1}(z), f_{2}(z) \in I_{p, q}(\alpha, A, B)$, by Theorem 2 , we obtain

$$
\begin{aligned}
& \sum_{n=1}^{\infty}\left((B+1)[n+p]_{q}\left(\alpha[n+p]_{q}-[p]_{q}\right)+(1+A)(1-\alpha)[p]_{q}^{2}\right)\left(\lambda\left|a_{n+p, 1}\right|+(1-\lambda)\left|a_{n+p, 2}\right|\right) \\
& =\lambda \sum_{n=1}^{\infty}\left((B+1)[n+p]_{q}\left(\alpha[n+p]_{q}-[p]_{q}\right)+(1+A)(1-\alpha)[p]_{q}^{2}\right)\left|a_{n+p, 1}\right| \\
& +(1-\lambda) \sum_{n=1}^{\infty}\left((B+1)[n+p]_{q}\left(\alpha[n+p]_{q}-[p]_{q}\right)+(1+A)(1-\alpha)[p]_{q}^{2}\right)\left|a_{n+p, 2}\right| \\
& \leq \lambda(A-B)(1-\alpha)[p]_{q}^{2}+(A-B)(1-\alpha)(1-\lambda)[p]_{q}^{2} \\
& =(A-B)(1-\alpha)[p]_{q}^{2} .
\end{aligned}
$$

This indicates that the function $H_{\lambda}(z)$ belongs to $I_{p, q}(\alpha, A, B)$. Now we complete the proof of Theorem 10.

Corollary 7. Let $\frac{[p]_{q}}{[p+1]_{q}} \leqq \alpha<1$ and

$$
f_{j}(z)=z^{p}-\sum_{n=1}^{\infty}\left|a_{p+n, j}\right| z^{p+n} \in I_{p, q}(\alpha, A, B) \quad(j=1,2, \cdots, l) .
$$

Then $F(z)=\sum_{j=1}^{l} \lambda_{j} f_{j}(z)\left(\lambda_{j} \geqq 0\right.$ and $\left.\sum_{j=1}^{l} \lambda_{j}=1\right)$ also belongs to $I_{p, q}(\alpha, A, B)$.

Theorem 11. Let $\frac{[p]_{q}}{[p+1]_{q}} \leqq \alpha<1$. If $f_{j}(z)=z^{p}-\sum_{n=1}^{\infty}\left|a_{p+n, j}\right| z^{p+n}$ belongs to $I_{p, q}(\alpha, A, B)$ $(j=1,2)$, then $Q_{m}(z)=\frac{(1-m) f_{1}(z)+(1+m) f_{2}(z)}{2}(-1 \leqq m \leqq 1)$ also belongs to $I_{p, q}(\alpha, A, B)$.

Proof of Theorem 11. Theorem 11 is proved using the similar arguments as in Theorem 10.

Author Contributions: Writing—original draft, B.W.; Writing—review \& editing, R.S. and J.-L.L. All authors have read and agreed to the published version of the manuscript.

Funding: This research received no external funding.

Institutional Review Board Statement: Not applicable.

Informed Consent Statement: Not applicable.

Data Availability Statement: Not applicable.

Acknowledgments: We would like to thank the reviewers for careful checking and suggestions.

Conflicts of Interest: The authors declare no conflict of interest.

\section{References}

1. Jackson, F.H. On q-defnite integrals. Quart. J. Pure Appl. Math. 1910, 41, 193-203.

2. Jackson, F.H. On $q$-functions and a certain difference operator. Trans. R. Soc. Edinb. 1908, 46, 64-72. [CrossRef]

3. Ismail, M.E.H.; Merkes, E.; Styer, D. A generalization of starlike functions. Complex Var. Theory Appl. 1990, 14, 77-84. [CrossRef]

4. Aral, A. On the generalized Picard and Gauss-Weierstrass singular integrals. J. Comput. Anal. Appl. 2006, 8, $249-261$.

5. Anastassiou, G.A.; Gal, S.G. Geometric and approximation properties of generalized singular integrals in the unit disk. J. Korean Math. Soc. 2006, 43, 425-443. [CrossRef]

6. Anastassiou, G.A.; Gal, S.G. Geometric and approximation properties of some singular integrals in the unit disk. J. Inequal. Appl. 2006, 2006, 1-19. [CrossRef]

7. Janowski, W. Some extremal problems for certain families of analytic functions. Ann. Pol. Math. 1973, 28, 297-326. [CrossRef]

8. Rehman, M.S.U.; Ahmad, Q.Z.; Srivastava, H.M.; Khan, N.; Darus, M.; Khan, B. Applications of higher-order $q$-derivatives to the subclass of $q$-starlike functions associated with the Janowski functions. AIMS Math. 2021, 6, 1110-1125. [CrossRef]

9. Srivastava, H.M.; Khan, B.; Khan, N.; Ahmad, Q.Z. Coefficient inequalities for $q$-starlike functions associated with the Janowski functions. Hokkaido Math. J. 2019, 48, 407-425. [CrossRef]

10. Srivastava, H.M.; Tahir, M.; Khan, B.; Ahmad, Q.Z.; Khan, N. Some general classes of $q$-starlike functions associated with the Janowski functions. Symmetry 2019, 11, 292.

11. Aldweby, H.; Darus, M. Coefficient estimates of classes of $q$-starlike and $q$-convex functions. Adv. Stud. Contemp. Math. 2016, $26,21-26$. 
12. Arif, M.; Srivastava, H.M.; Umar, S. Some applications of a $q$-analogue of the Ruscheweyh type operator for multivalent functions. Rev. Real Acad. Cienc. Exactas Fís. Nat. Ser. A Mat. 2019, 113, 1211-1221. [CrossRef]

13. Aouf, M.K.; Seoudy, T.M. Convolution properties for classes of bounded analytic functions with complex order defined by q-derivative operator. Rev. Real Acad. Cienc. Exactas Fís. Nat. Ser. A Mat. 2019, 113, 1279-1288. [CrossRef]

14. Aldweby, H.; Darus, M. Integral operator defined by $q$-analogue of Liu-Srivastava operator. Studia Univ. Babeş-Bolyai Ser. Math. 2013, 58, 529-537.

15. Mahmmod, S.; Sokół, J. New subclass of analytic functions in conical domain associated with Ruscheweyh $q$-differential operator. Results Math. 2017, 71, 1345-1357. [CrossRef]

16. Seoudy, T.M.; Aouf, M.K. Coefficient estimates of new classes of $q$-starlike and $q$-convex functions of complex order. J. Math. Inequal. 2016, 10, 135-145. [CrossRef]

17. Srivastava, H.M.; Bansal, D. Close-to-convexity of a certain family of $q$-Mittag-Leffler functions. J. Nonlinear Var. Anal. 2017, 1, 61-69.

18. Srivastava, H.M. Operators of basic (or $q$-) calculus and fractional $q$-calculus and their applications in geometric function theory of complex analysis. Iran. J. Sci. Technol. Trans. A Sci. 2020, 44, 327-344. [CrossRef]

19. Fekete, M.; Szegö, G. Eine Bemerkung Über ungerade schlichte Funktionen. J. Lond. Math. Soc. 1933, 8, 85-89. [CrossRef]

20. Srivastava, H.M.; Raza, N.; AbuJarad, E.S.A.; Srivastava, G.; AbuJarad, M.H. Fekete-Szegö inequality for classes of $(p ; q)$-starlike and $(p ; q)$-convex functions. Rev. Real Acad. Cienc. Exactas Fís Nat. Ser. A Mat. 2019, 113, 3563-3584. [CrossRef]

21. Ali, R.M.; Ravichandran, V.; Seenivasagan, N. Coefficient bounds for $p$-valent functions. Appl. Math. Comput. 2007, 187, 35-46. [CrossRef]

22. Keogh, F.R.; Merkes, E.P. A coefficient inequality for certain classes of analytic functions. Proc. Am. Math. Soc. 1969, 20, 8-12. [CrossRef]

23. Ma, W.C.; Minda, D. A unified treatment of some special classes of univalent functions. In Proceedings of the International Conference on Complex Analysis, Tianjin, China, 19-23 June 1992; pp. 157-169.

24. Prokhorov, D.V.; Szynal, J. Inverse coefficients for $(\alpha ; \beta)$-convex functions. Ann. Univ. Mariae Curie-Skłodowska Sect. 1981, $35,125-143$. 